\title{
CORE AND GLOBAL PROPERTIES OF EARLY-TYPE GALAXIES AND THEIR GLOBULAR CLUSTER SYSTEMS
}

\author{
Patrick CÔté and The ACS Virgo And Fornax Cluster Survey Teams \\ Herzberg Institute of Astrophysics, 5071 West Saanich Road, Victoria, BC, V9E 2E7, Canada \\ (Received June 16, 2010; Accepted August 11, 2010)
}

\begin{abstract}
The core and global properties of the early-type ("red sequence") galaxies in the Virgo and Fornax clusters are examined using high-quality HST/ACS imaging for 143 galaxies. Rather than dividing neatly into disparate populations having distinct formation and/or evolution histories, many of the core and global properties of these galaxies show smooth and systematic variations along the galaxy luminosity function. The few examples of the rare class of compact elliptical galaxies in our sample all show properties that are strongly suggestive of tidal stripping by massive galaxies; if so, then these systems should not be viewed as populating the low-luminosity extension of so-called "normal" elliptical sequences. These results demonstrate that complete and/or unbiased samples are a pre-requisite for identifying the physical mechanisms that gave rise to the early-type galaxies we observe locally, and how these mechanisms varied with mass and environment.
\end{abstract}

key words: galaxies: clusters: individual: name: Virgo; galaxies: elliptical and lenticular, cD; galaxies: nuclei; galaxies: star clusters

\section{INTRODUCTION}

An understanding of the physical processes that shaped the structure of galaxies we observe locally is a key goal of modern astrophysics. This goal, however, has proved challenging for early-type galaxies which are historically believed to exhibit puzzling structural "dichotomies" that suggest sharply disjointed formation processes. For instance, bright elliptical galaxies have been reported to divide - at roughly $M_{B} \approx-20.5$, corresponding to a stellar mass of $M_{*} \approx 2 \times 10^{11} \mathrm{M}_{\odot}$ into two distinct populations based on the logarithmic slope of their central surface brightness profiles (i.e., the "core/power - law dichotomy"; e.g., Ferrarese et al., 1994; Lauer et al., 1995). This apparent dichotomy has been interpreted as evidence for two distinct galaxy populations whose core properties are thought to result from either supermassive binary black hole evolution ("core" galaxies) or gas dissipation ("power-law" galaxies).

Meanwhile, at somewhat lower luminosities and masses (i.e., $M_{B} \approx-17.5$ and $M_{*} \approx 6 \times 10^{9} \mathrm{M}_{\odot}$ ), an abrupt change in central and global structure has been claimed to separate "normal" ellipticals (comprised of both "core" and "power-law" galaxies) from low-luminosity "dwarf" or "spheroidal" systems (e.g., Kormendy, 1985). It should be noted, however, that several subsequent studies have questioned the existence of both dichotomies, finding that at least some galaxies with intermediate properties may bridge the respective gaps (e.g., Jerjen \& Binggeli, 1997; Rest et al., 2001; Ravindranath et al., 2001; Graham et al., 2003; Graham \& Guzmán, 2003; Gavazzi et al., 2005).

\section{SAMPLE SELECTION AND ANALYSIS}

The ACS Virgo (Côté et al., 2004) and Fornax (Jordán et al., 2007a) Cluster Surveys are large HST imaging programs that were carried out, in part, to characterize these structural dichotomies using high-resolution, multi-color, homogeneous imaging for a carefully se- 


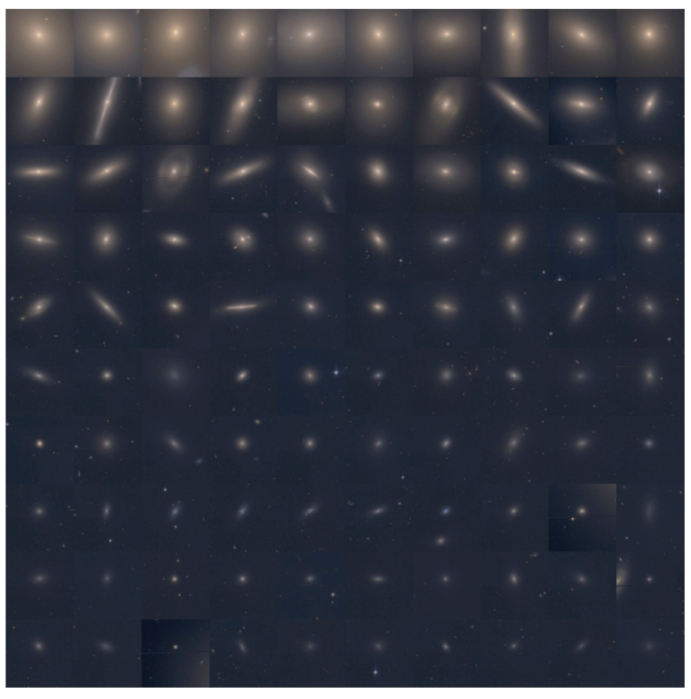

Fig 1.- Mosaic showing the 100 galaxies observed in the ACS Virgo Cluster Survey. These galaxies constitute a complete sample of early-type systems in the Virgo Cluster brighter than $M_{B} \approx-19$, and an unbiased sample of $54 \%$ of all Virgo early types down to $M_{B} \approx-15$. The corresponding sample in the Fornax Cluster is complete down to $M_{B} \approx$ -16.1 .

lected sample of early-type galaxies belonging to the two galaxy clusters nearest to the Milky Way (see Figure 1$)^{1}$. In order to shed light on the dichotomies mentioned above, the central and global properties of earlytype galaxies (e.g., E, S0, E/S0, S0/E, or their "dwarf" counterparts; Binggeli et al., 1985; Ferguson, 1989) were derived simultaneously by parameterizing the surface brightness profiles - derived from ACS imaging and supplemented with ground-based photometry from the literature or from SDSS — with the family of Sérsic models (e.g., Sérsic, 1968; Caon et al., 1993, 1994; Graham et al., 2003). A secondary goal of the surveys (see §3.3) was to characterize the properties of globular cluster systems for a large sample of early-type galaxies that span a wide range in galaxy luminosity and mass, thereby extending pioneering HST archival studies of globular clusters in early-type environments (Gebhardt \& Kissler-Patig, 1999; Kundu \& Whitmore, 2001; Larsen et al., 2001).

At this point, a word on the sample selection is in

\footnotetext{
${ }^{1}$ For more information on these HST programs, see: https://www.astrosci.ca/users/VCSFCS
}

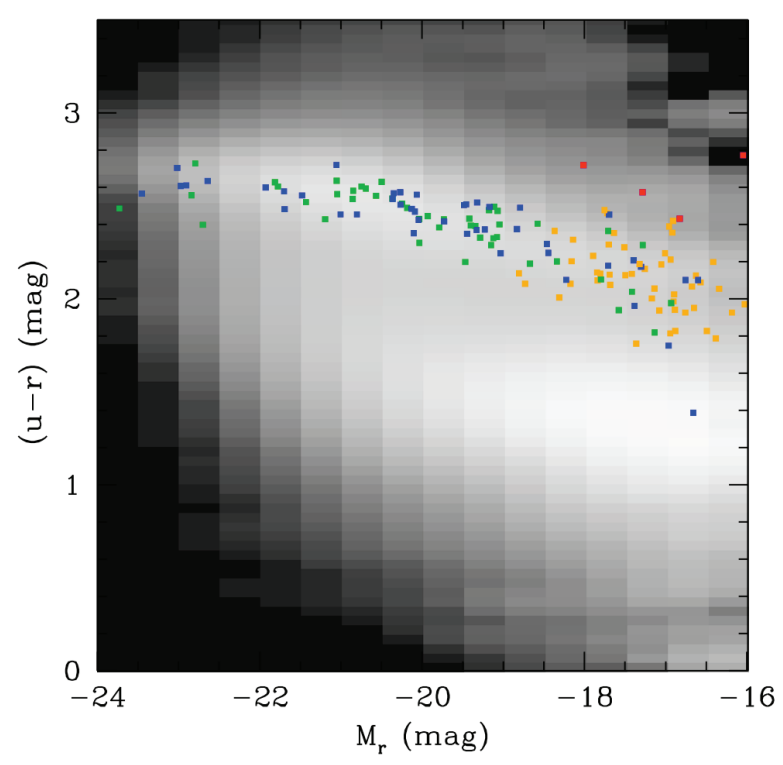

Fig 2.- Distribution of ACS Virgo and ACS Fornax Cluster Survey galaxies in the $M_{r}-(u-r)$ colormagnitude diagram. Note that these galaxies fall along the red sequence in the color-magnitude diagram. The grayscale image shows the distribution for a low-redshift sample of galaxies from the SDSS (Baldry et al., 2004). Although there is disagreement over the exact morphological classifications for many of the Virgo and Fornax galaxies, they have been tentatively divided into Es, S0s and "dwarfs" (blue, green and orange symbols, respectively). Red symbols show the rare class of "compact ellipticals" in our sample.

order. In many past studies, galaxies were selected on the basis of morphology (e.g., choosing pure samples of Es, S0s, or dwarfs - often by relying on morphological classifications from early photographic resources such as the RC3). Since our comparison of morphological classifications for Virgo and Fornax galaxies taken from various sources often showed poor agreement particularly at intermediate luminosities where the distinction between E and S0s (see also Emsellem et al. 2007) and between "giants" and "dwarfs" is not always obvious from visual inspection - it was decided to focus on the ensemble of early type galaxies. As Figure 2 shows, this broad morphological selection effectively isolates those galaxies that define the local "red sequence" in the color-magnitude diagrams of these two clusters. Thus, the sample selection used here roughly mirrors the one that is widely used in the study of much 

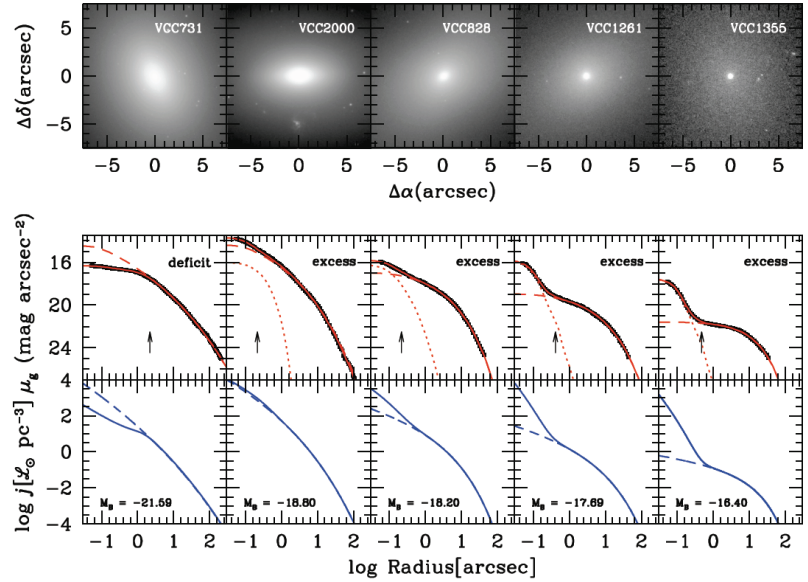

Fig 3. - Five red-sequence galaxies from the ACS Virgo Cluster Survey chosen to illustrate the continuities in central and global structure. (Upper Row) ACS/F475W images showing the inner $15^{\prime \prime} \times 15^{\prime \prime}$ $(\approx 1.2 \mathrm{kpc} \times 1.2 \mathrm{kpc}$ ) for each galaxy. (Middle Row) Azimuthally-averaged surface brightness profiles and the best-fit double Sérsic models; the dotted and dashed curves show the nuclear and global components, respectively. [For the brightest galaxy, the best-fit "core-Sérsic" model is shown.] The arrow in each panel is drawn at $2 \%$ of the effective radius of the galaxy. (Lower Row) Deprojected, luminosity density profiles for the same five galaxies (from Glass et al., 2010). The solid curves show the deprojected profiles corresponding to the solid curves shown in the middle row. Dashed curves show the profiles corresponding to the inward extrapolation of the Sérsic models that best fit the outer profile (i.e., the main body of the galaxy itself).

more distant galaxy populations.

\section{RESULTS}

3.1. Central Surface Brightness Profiles: From a Luminosity "Deficit" to "Excess"

Figure 3 shows that the azimuthally-averaged surface brightness profiles of early-type galaxies can be parameterized accurately over a wide range in radius $(z 3$ decades) by Sérsic (1968) models (see Ferrarese et al., 2006a, b; Côté et al., 2006, and earlier studies by Caon et al., 1993, 1994; Jerjen \& Binggeli, 1997; Graham \& Guzman, 2003; Gavazzi et al., 2005). This applies not just to luminous "giants" (with Sérsic indices of $n \sim 4$ )

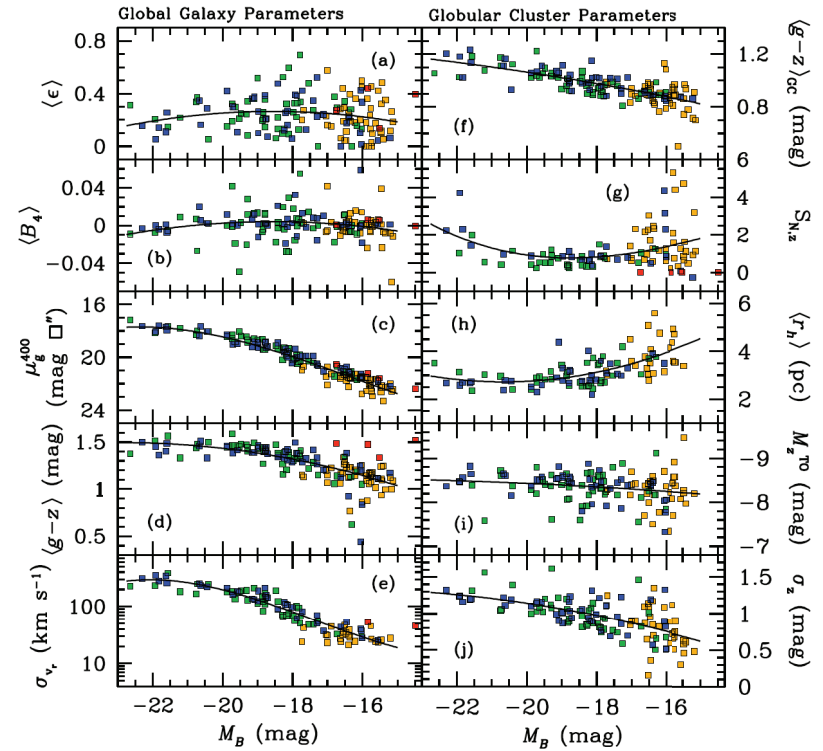

Fig 4.- Global properties for galaxies from the ACS Virgo and Fornax Cluster Surveys. The panels on the left (a-e) show parameters relating to the galaxies themselves while those on the right $(f-j)$ show the global properties of their associated globular cluster systems. Galaxies have been divided by morphology as in Figure 2. The red symbols show the "compact ellipticals" which are outliers in Figure 2. The smooth curves show polynomials fitted to each parameter, highlighting the continuities along the red sequence luminosity function.

and faint "dwarfs" $(n \sim 1)$, but also to galaxies with intermediate luminosities.

An important qualification is that there are systematic deviations in the innermost regions $\left(R \lesssim 0.02 R_{e}\right)$ of most galaxies. Those brighter than $M_{B} \sim-20.5$ $\left(M_{*} \sim 10^{11} \mathrm{M}_{\odot}\right)$ show central luminosity deficits with respect to inward extrapolations of Sérsic models, possibly due to core evacuation caused by coalescing black hole binaries (e.g. Ebisuzaki et al., 1991; Faber et al., 1997; Milosavljević \& Merritt, 2001). By contrast, galaxies fainter than $M_{B} \sim-19.5\left(M_{*} \sim 10^{10.6} \mathrm{M}_{\odot}\right)$ usually show central luminosity excesses, or nuclei (Ferrarese et al., 2006b; Côté et al., 2006) ${ }^{2}$ that may in some cases be the signature of star formation resulting from gas inflows during mergers or accretions (Mihos \& Hernquist, 1994; Côté et al., 2007; Hopkins et al., 2009; Kormendy et al., 2009), although other processes,

${ }^{2}$ This result has recently been confirmed by Kormendy et al. (2009), who re-analyzed a subset (40/100) of the ACS Virgo Cluster Survey galaxies. 
such as nucleus growth from globular clusters that have been drawn to the galaxy center by dynamical friction (Tremaine et al., 1975), may also play a role. In any case, these deviations typically amount to less than one percent of the total galaxy luminosity and they are confined to within $\sim 1 \%$ of the radial range over which Sérsic models fit with high accuracy. Thus, these central deviations do not influence the global structural parameters.

\subsection{Global Properties}

Figure 4 confirms that, although the global properties of the galaxies do indeed vary along the luminosity function, they do not do divide into discrete, mutually exclusive populations. For instance, the left panels of this figure plot the variation with absolute magnitude of several basic global parameters: mean ellipticity, mean isophote shape, $g$-band surface brightness measured at a fixed physical scale of $400 \mathrm{pc}$, mean $(g-z)$ color, and velocity dispersion measured inside $\mathrm{R}_{e} / 8$. The smooth curves drawn in each panel show low-order polynomial fits, illustrating the continuities along the luminosity function. This exercise demonstrates the importance of sample selection: i.e., had one simply selected samples of bright ("normal") ellipticals and faint ("dwarf") galaxies, it would be tempting to conclude that the two samples are fundamentally distinct in their properties.

\subsection{Globular Cluster Properties Along the Luminos- ity Function}

As mentioned in $\S 1$, a second motivation of the ACS Virgo and Fornax Cluster Surveys was a characterization of the properties of globular clusters in early-type galaxies. The right panels of Figure 4 show the variation along the galaxy luminosity function of several important observables for the globular clusters in these galaxies, taken from the studies of Jordán et al. (2005, 2007b), Peng et al. (2006, 2008), Villegas et al. (2010) and Masters et al. (2010). From top to bottom, these panels show the mean globular cluster color, specific frequency, mean half-light radius, and the "turnover" magnitude and dispersion of the globular cluster luminosity function. In almost every case, there is a gradual and systematic variation along the red sequence luminosity function, contrary to what would be expected if there were sharp discontinuities in the physical processes that shaped these galaxies.

\subsection{The Case of the Compact Ellipticals}

Although Figures 2 and 4 show smooth trends from the brightest elliptical galaxies down to the regime of "dwarf" galaxies (i.e., those systems usually denoted by the $\mathrm{dE}, \mathrm{dS} 0, \mathrm{dSph}$ or Sph nomenclature), there is a handful of galaxies that do seem to depart from the sequences defined by the majority of early-type galaxies. These four galaxies, which are plotted in red in both figures, are examples of the rare class of "compact ellipticals".

Like M32, the proto-type for this class, each of these galaxies is found close to a much more massive companion. Moreover, all have some unusual properties that are qualitatively consistent with those expected for objects that have undergone substantial tidal stripping (Faber, 1973; Bekki et al., 2001; Chilingarian et al., 2009): e.g., compact size, high surface brightness, unusually red color and reduced GC specific frequency. These results suggest that compact ellipticals like M32 should not be viewed as low-luminosity analogs of "giant E" galaxies, as has sometimes been claimed (e.g., Kormendy, 1985; Kormendy et al., 2009), but, rather, as unusual objects whose extreme properties are the signature of an atypical formation channel, in which one of the many physical processes (e.g., merging, stripping, harassment, dissipation, etc.) acting on galaxies has been dominant in their evolution (Chilingarian et al., 2009; Price et al., 2009). This interpretation would be consistent with the observation of Bender et al. (1992) that: "The rarity of such galaxies [cEs] suggests that this does not happen often during galaxy formation."

\section{DIRECTIONS FOR FUTURE RESEARCH}

A number of conclusions can be drawn from the above discussion. First, the importance of a well-defined, objective galaxy selection function can scarcely be overestimated; while morphology will always play a role in the study of galaxy evolution, it seems preferable in the future to use quantitative selection criteria (such as location in the galaxy color-magnitude diagram; see Figure 2) to isolate complete and/or unbiased samples that can be then used to determine what morphology may be telling us about the relative balance of the physical properties driving galaxy evolution, rather than the other way around. Second, it will be important to extend the imaging surveys described here to lower masses and bluer colors (= later types) with high lev- 
els of completeness. Two large imaging surveys are now underway that should provide important steps forward in this area: (1) the ACS Coma Cluster Survey on HST (PI = Carter) and; (2) the Next Generation Virgo Cluster Survey on CFHT (PI= Ferrarese). The latter survey, which should be completed in 2012, is currently surveying the entire Virgo Cluster in ugiz down to a point-source depth of $g_{A B} \simeq 25.7(10 \sigma)$. It will enable a more complete characterization of galaxy scaling relations - as well as those of their globular cluster systems - from the level of brightest cluster members down to dwarf galaxies with luminosities approaching those of Galactic dSphs.

\section{ACKNOWLEDGEMENTS}

I thank my collaborators from the ACS Virgo and Fornax Surveys for allowing me to present some results in advance of publication, and Profs. Sukyoung Yi and Suk-Jin Yoon for their extraordinary hospitality during this superb workshop.

\section{REFERENCES}

Baldry, I. K., et al., 2004, Quantifying the Bimodal Color-Magnitude Distribution of Galaxies, ApJ, 600,681

Binggeli, B., Sandage, A., \& Tammann, G. A., 1985, Studies of the Virgo Cluster. II - A Catalog of 2096 Galaxies in the Virgo Cluster Area, AJ, 90, 1681

Bekki, K., et al., 2001, A New Formation Model for M32: A Threshed Early-Type Spiral Galaxy?, ApJ, 557, L39

Bender, R., Burstein, D., \& Faber, S. M., 1992, Dynamically Hot Galaxies. I - Structural Properties, ApJ, 399, 462

Caon, N., Capaccioli, M., \& D'Onofrio, M., 1993, On the Shape of the Light Profiles of Early Type Galaxies, MNRAS, 265, 1013

Caon, N., Capaccioli, M., \& D'Onofrio, M., 1994, 'Global Mapping' B-Band Photometry of a Complete Sample of Fornax and Virgo Early-Type Galaxies, A\&AS, 106, 199

Chilingarian, I., et al., 2009, A Population of Compact Elliptical Galaxies Detected with the Virtual Observatory, Science, 326, 1379

Côté, P., et al., 2004, The ACS Virgo Cluster Survey. I. Introduction to the Survey, ApJS, 153, 223

Côté, P., et al., 2006, The ACS Virgo Cluster Survey. VIII. The Nuclei of Early-Type Galaxies, ApJS, 165,57
Côté, P., et al., 2007, The ACS Fornax Cluster Survey. II. The Central Brightness Profiles of EarlyType Galaxies: A Characteristic Radius on Nuclear Scales and the Transition from Central Luminosity Deficit to Excess, ApJ, 671, 1456

Ebisuzaki, T., Makino, J., \& Okumura, S. K., 1991, Merging of Two Galaxies with Central Black Holes, Nature, 354, 212

Emsellem, E., et al., 2007, The SAURON project - IX. A Kinematic Classification for Early-Type Galaxies, MNRAS, 379, 401

Faber, S. M., 1973, Tidal Origin of Elliptical Galaxies of High Surface Brightness, ApJ, 179, 423

Faber, S. M., et al., 1997, The Centers of Early-Type Galaxies with HST. IV. Central Parameter Relations, AJ, 114, 1771

Ferguson, H. C., 1989, Population Studies in Groups and Clusters of Galaxies. II - A Catalog of Galaxies in the Central $3.5 \mathrm{deg}$ of the Fornax Cluster, AJ, 98, 367

Ferrarese, L., et al., 1994, Hubble Space Telescope Photometry of the Central Regions of Virgo Cluster Elliptical Galaxies. 3: Brightness Profiles, AJ, 108, 1598

Ferrarese, L., et al., 2006a, The ACS Virgo Cluster Survey. VI. Isophotal Analysis and the Structure of Early-Type Galaxies, ApJS, 164, 334

Ferrarese, L., et al., 2006b, A Fundamental Relation between Compact Stellar Nuclei, Supermassive Black Holes, and Their Host Galaxies, ApJ, 644, L21

Gavazzi, G., Donati, A., Cucciati, O., Sabatini, S., Boselli, A., Davies, J., \& Zibetti, S., 2005, The Structure of Elliptical Galaxies in the Virgo Cluster. Results from the INT Wide Field Survey, A\&A, 430, 411

Gebhardt, K. \& Kissler-Patig, M., 1999, Globular Cluster Systems. I. V-I Color Distributions, AJ, 118,1526

Glass, L., et al., 2010, ApJ, submitted.

Graham, A. W., et al., 2003, A New Empirical Model for the Structural Analysis of Early-Type Galaxies, and a Critical Review of the Nuker Model, AJ, 125, 2951

Graham, A. W. \& Guzmán, R., 2003, HST Photometry of Dwarf Elliptical Galaxies in Coma, and an Explanation for the Alleged Structural Dichotomy between Dwarf and Bright Elliptical Galaxies, AJ, 125, 2936

Hopkins, P. F., et al., 2009, Dissipation and Extra Light in Galactic Nuclei. IV. Evolution in the Scaling Relations of Spheroids, ApJ, 691, 1424 
Jerjen, H. \& Binggeli, B., 1997, The Nature of Elliptical Galaxies; 2nd Stromlo Symposium, 116, 239

Jordán, A., et al., 2005, The ACS Virgo Cluster Survey. X. Half-Light Radii of Globular Clusters in Early-Type Galaxies: Environmental Dependencies and a Standard Ruler for Distance Estimation, ApJ, 634, 1002

Jordán, A., et al., 2007a, The ACS Fornax Cluster Survey. I. Introduction to the Survey and Data Reduction Procedures, ApJS, 169, 213

Jordán, A., et al., 2007b, The ACS Virgo Cluster Survey. XII. The Luminosity Function of Globular Clusters in Early-Type Galaxies, ApJS, 171, 101

Kormendy, J., 1985, Families of Ellipsoidal Stellar Systems and the Formation of Dwarf Elliptical Galaxies, ApJ, 295, 73

Kormendy, J., Fisher, D. B., Cornell, M. E., \& Bender, R., 2009, Structure and Formation of Elliptical and Spheroidal Galaxies, ApJS, 182, 216

Kundu, A. \& Whitmore, B. C., 2001, New Insights from HST Studies of Globular Cluster Systems. I. Colors, Distances, and Specific Frequencies of 28 Elliptical Galaxies, AJ, 121, 2950

Larsen, S. S., Brodie, J. P., Huchra, J. P., Forbes, D. A., \& Grillmair, C. J., 2001, Properties of Globular Cluster Systems in Nearby Early-Type Galaxies, AJ, 121, 2974

Lauer, T. R., et al., 1995, The Centers of Early-Type Galaxies with HST. I. An Observational Survey, AJ, 110, 2622

Masters, K. L., et al., 2010, The Advanced Camera for Surveys Fornax Cluster Survey. VII. HalfLight Radii of Globular Clusters in Early-type Galaxies, ApJ, 715, 1419

Mihos, J. C. \& Hernquist, L., 1994, Triggering of Starbursts in Galaxies by Minor Mergers, ApJ, 425, 13

Milosavljević, M. \& Merritt, D., 2001, Formation of Galactic Nuclei, ApJ, 563, 34

Peng, E. W., et al., 2006, The ACS Virgo Cluster Survey. IX. The Color Distributions of Globular Cluster Systems in Early-Type Galaxies, ApJ, 639,95

Peng, E. W., et al., 2008, The ACS Virgo Cluster Survey. XV. The Formation Efficiencies of Globular Clusters in Early-Type Galaxies: The Effects of Mass and Environment, ApJ, 681, 197

Price, J., et al., 2009, The HST/ACS Coma Cluster Survey - V. Compact Stellar Systems in the Coma Cluster, MNRAS, 397, 1816
Ravindranath, S., Ho, L. C., Peng, C. Y., Filippenko, A. V., \& Sargent, W. L. W., 2001, Central Structural Parameters of Early-Type Galaxies as Viewed with Nicmos on the Hubble Space Telescope, AJ, 122, 653

Rest, A., van den Bosch, F. C., Jaffe, W., Tran, H., Tsvetanov, Z., Ford, H. C., Davies, J., \& Schafer, J., 2001, WFPC2 Images of the Central Regions of Early-Type Galaxies. I. The Data, AJ, 121, 2431

Sersic, J. L., 1968, Cordoba, Argentina: Observatorio Astronomico, 1968

Tremaine, S. D., Ostriker, J. P., \& Spitzer, L., Jr., 1975, The Formation of the Nuclei of Galaxies. I - M31, ApJ, 196, 407

Villegas, D., et al., 2010, The ACS Fornax Cluster Survey. VIII. The Luminosity Function of Globular Clusters in Virgo and Fornax Early-Type Galaxies and Its Use as a Distance Indicator, ApJ, 717, 603 